\title{
A Study of the Interaction between Oral Streptococci and Hard Surfaces
}

\author{
By PAUL R. RUTTER AND ANTHONY ABBOTT \\ Gibbs Dental Research, Unilever Research, Isleworth Laboratory, \\ 455 London Road, Isleworth, Middlesex
}

(Received 9 June 1977; revised 23 November 1977)

\begin{abstract}
A rotating disc method was used to compare the tendencies of two oral streptococci to deposit on to glass and polystyrene surfaces from electrolyte solutions of varying ionic strength. Streptococcus salivarius had a greater tendency to deposit than had Streptococcus mitior under these conditions. In addition to the balance of van der Waals' forces of attraction and electrostatic forces of repulsion, it is suggested that the adsorption to the glass and polystyrene surfaces of material present in the outer layers of the cell wall could play a significant part in the deposition of $S$. salivarius.
\end{abstract}

\section{INTRODUCTION}

Bacteria accumulate on hard surfaces in a number of very different natural environments ranging from sand grains in sea water to tooth surfaces in the oral cavity.

With marine bacteria, Marshall, Stout \& Mitchell (1971) showed that sorption of organisms to surfaces involved an initial reversible phase and a time-dependent irreversible phase. The reversible phase probably depended on a balance between the electrical doublelayer repulsion energies and the van der Waals' attractive energies of the bacteria and the substrate. The irreversible phase was thought to involve polymer bridging from the bacterial surface to the substrate.

The initial interaction between bacteria and the substrate, referred to here as 'deposition', is controlled by the physical properties of the bacteria and the substrate surface and can be studied using the principles employed in colloid science. To study deposition it is necessary to know the rate at which particles are supplied to the surface and the number of collisions which result in capture. The well-defined hydrodynamics associated with a disc rotating in a liquid medium make it a useful method of assessing particle deposition (Levich, 1962; Marshal \& Kitchener, 1966). The present study investigates the tendencies of two organisms, which accumulate to different extents on teeth in the oral cavity (Gibbons $\&$ van Houte, 1973), to deposit on to glass and polystyrene surfaces from salt solutions.

\section{METHODS}

Organisms. The bacteria used in this investigation were Streptococcus mitior (B. Guggenheim strain omz 44 23.11.70) and Streptococcus salivarius B128 (from George Bowden, 1974) isolated from the oral cavity. The organisms were maintained on blood agar plates. Culture characteristics were checked using the physiological tests outlined by Hardie \& Bowden (1976). For the experiments, $18 \mathrm{~h}$ stationary phase batch cultures were used. The cells were grown aerobically at $37^{\circ} \mathrm{C}$ in a defined medium recommended by Bowden (personal communication) supplemented with $0.019 \mathrm{M}-\mathrm{Na}_{2} \mathrm{CO}_{3}$. They were harvested by centrifugation $(3000 \mathrm{~g}, 30 \mathrm{~min})$ and resuspended in sterile distilled water to $1 / 50$ of the original culture volume. No washing stage was used so as to avoid the possibility of removing material associated with the wall. This heavy 
suspension was distributed in glass tubes for freeze drying on an Edwards High Vacuum Speedivac 5PS apparatus.

Cell suspensions. Bacterial suspensions were prepared by reconstituting freeze-dried organisms in the appropriate saline solution, dispersing them by sonication for $30 \mathrm{~s}$ (maximum amplitude, MSE $100 \mathrm{~W}$ ultrasonic disintegrator) and adjusting the absorbance of the suspension to about 0.2 at $570 \mathrm{~nm}$ (Unicam SP600 spectrophotometer). Suspensions of trypsin-digested cells were also used. These cells had been reconstituted into a 50/50 mixture of $0.15 \mathrm{M}$-saline and $0.1 \mathrm{M}$-phosphate buffer $\mathrm{pH} 7.8$ containing trypsin from beef pancreas (BDH) at $1 \mathrm{mg} \mathrm{ml}^{-1}$ (approx. 0.0005 Anson units $\mathrm{ml}^{-1}$ ) and incubated for $18 \mathrm{~h}$ at $37^{\circ} \mathrm{C}$. The cells were harvested and washed in the appropriate saline solution before resuspending and adjusting to an absorbance of about $0 \cdot 2$ at $570 \mathrm{~nm}$.

Glass. All the glass substrates used for deposition studies were degreased in acetone and absolute ethanol before soaking in chromic acid. The glass was thoroughly rinsed in double-distilled water from an all-glass still before use.

Polystyrene. Polystyrene surfaces were prepared by coating glass coverslips. The coverslips were cleaned by boiling in $0.5 \%(\mathrm{v} / \mathrm{v})$ Decon 90 surfactant for $1 \mathrm{~h}$ and were then rinsed in condensing steam for $1 \mathrm{~h}$ and dried under vacuum. They were then boiled in a $5 \%(\mathrm{w} / \mathrm{v})$ solution of polystyrene in toluene for $2 \mathrm{~h}$ after which the toluene solution was allowed to cool and the coverslips were slowly withdrawn leaving a thin film of polystyrene on the glass. The presence of a polystyrene film was confirmed by multiple internal reflected infrared and was shown to be about $100 \mathrm{~nm}$ thick by scanning electron microscopy.

Polystyrene latex. The polystyrene latex particles were prepared in our laboratory by the method of Goodwin et al. (1973).

Electrophoretic mobility. The electrophoretic mobilities of the bacteria were determined for various conditions of $\mathrm{pH}$ and ionic strength using a Rank microelectrophoresis apparatus. A thin-walled, narrow bore, cylindrical cell with platinum electrodes was used at room temperature under dark-ground illumination.

Qualitative assessment of deposition by sedimentation. Preliminary deposition experiments were carried out using a sedimentation technique (Fletcher, 1976). Bacterial suspensions $(15 \mathrm{ml})$ in different saline concentrations were placed in $9 \mathrm{~cm}$ polystyrene Petri dishes which contained shortened glass microscope slides. Deposition was allowed to occur over $2 \mathrm{~h}$. The suspensions were then discarded and the glass and polystyrene substrates were rinsed gently with distilled water. A qualitative assessment of deposition was made by staining the substrates with $1 \%(\mathrm{w} / \mathrm{v})$ crystal violet, rinsing off the excess stain with distilled water, and measuring the amount of stained deposit by strapping the substrate to the outside of a spectrophotometer cuvette holder and reading the absorbance at $570 \mathrm{~nm}$.

Deposition at the surface of a rotating disc. A spinning disc apparatus similar to that described by Marshal \& Kitchener (1966) was used. The basic unit consisted of a Perspex disc $5.5 \mathrm{~cm}$ in diameter. The disc was conically shaped to a height of $2.5 \mathrm{~cm}$ from the base and was screwed to a shaft which carried a socket. The socket enabled the shaft to be secured to the main drive shaft of a constant speed motor. The drive unit was supported by a ball race which prevented any lateral motion. A nylon coupling was placed between the drive shaft and the motor to prevent the transmission of vibrations from the motor to the disc. Suspensions $(40 \mathrm{ml})$ with different salt concentrations were sonicated $(7 \mu \mathrm{m}$ amplitude) for $10 \mathrm{~min}$ to disperse the bacteria, and then immediately placed in a $12.5 \mathrm{~cm}$ crystallizing dish which was on a laboratory jack beneath the disc. The dish was moved into position to immerse the rotating disc. The time taken to transfer the suspension, after sonication, to the deposition apparatus was less than $1 \mathrm{~min}$. Samples were taken to count the initial number of single cells immediately after sonication, counts being made on a Hawksley haemocytometer $(0.02 \mathrm{~mm})$. Clean glass coverslips and coverslips covered with thin polystyrene films were used as deposition surfaces and were attached to a recess in the base of the rotating disc by a trace of Edwards high vacuum silicone grease. The disc was spun at $180 \mathrm{rev}$. $\mathrm{min}^{-1}$ at $20^{\circ} \mathrm{C}$ for $10 \mathrm{~min}$ in the suspension. It was then removed and rinsed gently with $50 \mathrm{ml}$ of the suspending fluid. The coverslip was separated from the disc and the deposit was stained with $1 \%(\mathrm{w} / \mathrm{v})$ crystal violet. An optical microscope was used to count the deposited single bacteria in at least six separate fields.

\section{RESULTS}

\section{Electrophoretic mobility}

Table 1 shows the dependence on ionic strength of the electrophoretic mobilities of the two species of bacteria and the trypsin-treated bacteria. Trypsin treatment seems to have had little effect on the electrophoretic mobilities of either species. 
Table 1. Effect of ionic strength on electrophoretic mobilities of S. mitior and $S$. salivarius with and without trypsin treatment

The $\mathrm{pH}$ of the unbuffered suspensions was between $\mathrm{pH} 5.9$ and $6 \cdot 2$.

$\begin{array}{lccc}\text { Species } & \begin{array}{c}\text { Trypsin } \\ \text { treatment }\end{array} & \begin{array}{c}\mathrm{NaCl} \text { concn } \\ (\mathrm{mM})\end{array} & \begin{array}{c}10^{8} \times \text { Mobility } \\ \left(\mathrm{m}^{2} \mathrm{~V}^{-1} \mathrm{~s}^{-1}\right)\end{array} \\ \text { S. mitior } & + & 1 & -2 \cdot 732 \\ & & 10 & -2 \cdot 113 \\ \text { S. mitior } & - & 50 & -1 \cdot 474 \\ & 1 & -2 \cdot 549 \\ \text { S. salivarius } & + & 10 & -1.990 \\ & & 50 & -1 \cdot 157 \\ \text { S. salivarius } & 1 & -2 \cdot 445 \\ & - & 10 & -1 \cdot 623 \\ \text { Polystyrene } & & 1 & -1 \cdot 229 \\ \text { latex particles } & & 10 & -1 \cdot 747 \\ & & 50 & -1 \cdot 825 \\ & & 1 & -1 \cdot 234 \\ & & 10 & -2 \cdot 140 \\ & & 50 & -2 \cdot 140 \\ & & & -1 \cdot 124\end{array}$

Deposition by sedimentation

Figure 1 shows the variation with ionic strength of the deposition by sedimentation of the two species on to glass and polystyrene. The deposition of the species on to both substrates showed a dependence on ionic strength. Maximum deposition appears to have occurred at an ionic strength of between 0.03 and $0.1 \mathrm{M}$ for both organisms to both surfaces. There was no evidence of a charge reversal at this point. At ionic strengths above $0 \cdot 1 \mathrm{M}$ a reduction of deposition was observed in all cases.

\section{Deposition at the surface of a rotating disc}

Effect of ionic strength on bacterial deposition on to glass. The influence of ionic strength, determined by sodium chloride concentration, on bacterial deposition on to glass is represented by a plot of the logarithm of the stability ratio, $\log W$, of the suspension against the logarithm of the ionic strength, $\log I$ (Figs 2 and 3). The stability ratio of the suspension, $W$, is the total number of collisions divided by the number which result in capture. The total number of collisions is given by the Levich equation (Hull \& Kitchener, 1969):

$$
\frac{\mathrm{d} N}{\mathrm{~d} t}=0.62 D^{\frac{2}{3}} \nu^{-\frac{1}{6}} \omega^{\frac{1}{2}} N_{0}
$$

where $\nu$ is the kinematic viscosity, $\omega$ is the angular velocity of the disc, $N_{0}$ is the concentration of single particles in the initial suspension, and $D=k T / 6 \pi \eta a$ where $\eta$ is the absolute viscosity and $a$ is the radius of the particle. If the number of particles captured by the disc is $N_{\mathrm{d}}$, then

$$
W=\frac{\frac{\mathrm{d} N}{\mathrm{~d} t} \cdot t}{N_{\mathrm{d}}}
$$

where $t(\mathrm{~s})$ is the time of rotation of the disc. In our calculations of $W$ we assumed the diameters of $S$. salivarius and $S$. mitior to be $0.8 \mu \mathrm{m}$. Some $S$. mitior cultures tended to be more heterogeneous with respect to size, occasionally being smaller than $0.8 \mu \mathrm{m}$. This would increase $W$ and increase the observed difference in deposition between the two species. 


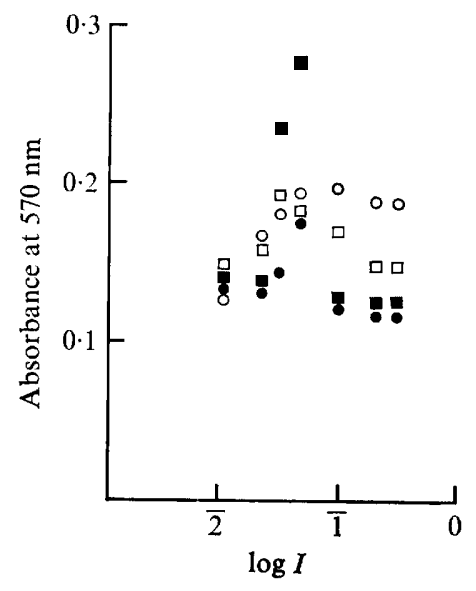

Fig. 1. Effect of ionic strength $(I)$ on the deposition by sedimentation of $S$. mitior $(\bigcirc, \square)$ and $S$. salivarius $(O, \boldsymbol{D})$ on to glass $(O, \Theta)$ and polystyrene $(\square, \square)$. Each point is the average of at least 12 values (S.D. $=0.016$ ).

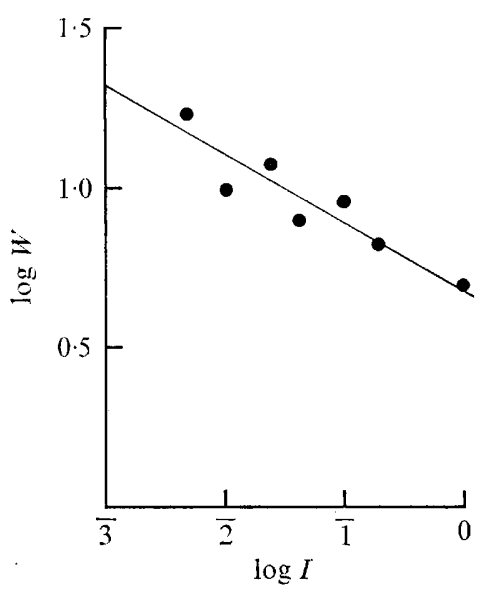

Fig. 2

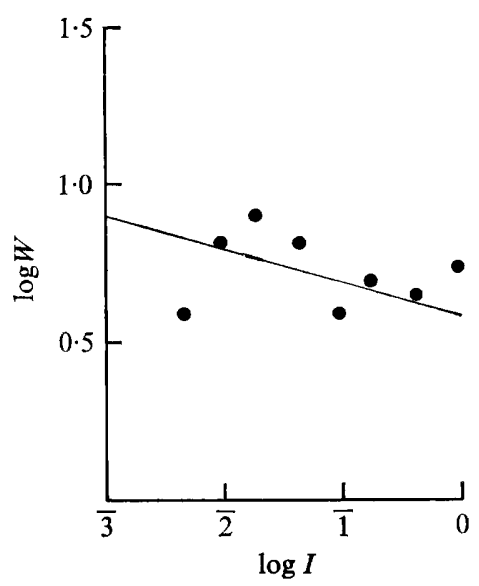

Fig. 3

Fig. 2. Effect of ionic strength on the deposition of $S$. mitior on to glass on the rotating disc. Each point is the average of at least 4 values. The line was fitted by computer from a total of 60 values: $\log W=1.26-0.195 \log I$; correlation coefficient $=0.51$.

Fig. 3. Effect of ionic strength on the deposition of $S$. salivarius on to glass on the rotating disc. Each point is the average of at least 4 values. The line was fitted by computer from a total of 70 values: $\log W=0.92-0.132 \log I$; correlation coefficient $=0.30$.

Aggregation during the course of the experiment tended to reduce the single particle concentration in the suspension with time. This reduces the theoretical calculated deposition. However, calculation shows that this had little effect on $W$ owing to the relatively low suspension concentrations and the short deposition times. Both species appeared to show a dependence on ionic strength in their tendencies to deposit. The lower gradient for the $S$. salivarius plot suggests a lesser dependence on ionic strength.

When $\mathrm{Na}_{2} \mathrm{SO}_{4}$ was used to vary the ionic strength (Fig. 4), both species showed a peak in their deposition capabilities at an ionic strength of about $0.05 \mathrm{M}$. Streptococcus salivarius exhibited a greater tendency to deposit on to glass at this ionic strength than did S. mitior. 


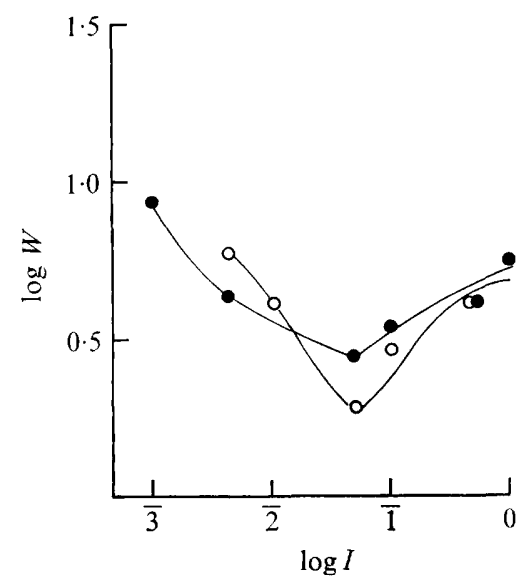

Fig. 4

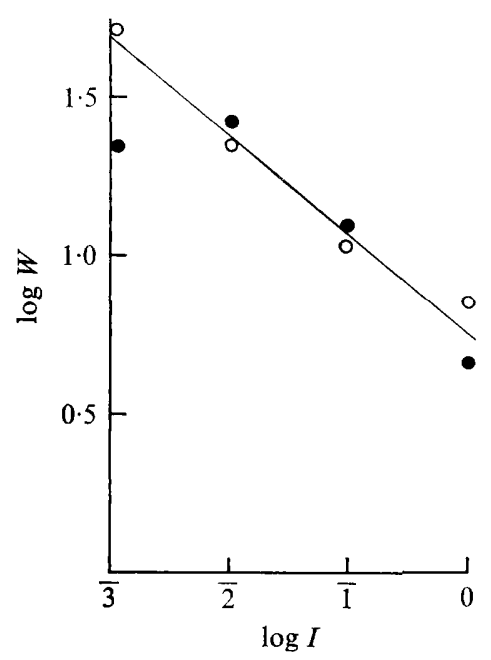

Fig. 5

Fig. 4. Effect of ionic strength, adjusted using $\mathrm{Na}_{2} \mathrm{SO}_{4}$, on the deposition of $S$. salivarius (O) and $S$. mitior ( ) on to glass on the rotating disc. Each point is the average of at least 4 values.

Fig. 5. Effect of ionic strength on the deposition of trypsin-treated $S$. salivarius $(O)$ and trypsintreated $S$. mitior (O) on to glass on the rotating disc. Each point is the average of at least 4 values.

Influence of trypsin treatment of bacteria on the relationship between ionic strength and deposition on to glass. The effect of trypsin treatment on bacterial deposition on to glass is shown in Fig. 5. After trypsin treatment both species showed a greater dependence on ionic strength. The trypsin appears to have had a relatively greater effect on $S$. salivarius.

Electron micrographs of treated and untreated bacteria suggest that $S$. salivarius has a fuzzy layer around the cell which is largely removed by trypsin treatment. However, $S$. mitior appears to have a thinner fuzzy layer which is little affected by trypsin treatment.

Effect of ionic strength on bacterial deposition on to polystyrene. The effect of ionic strength on the deposition of $S$. mitior and $S$. salivarius on to polystyrene is shown in Fig. 6 . The deposition of both species was influenced by ionic strength. Streptococcus salivarius had a greater tendency to deposit than had $S$. mitior, but both exhibited a peak at around 0.03 to $0.05 \mathrm{M}$.

Influence of trypsin treatment of bacteria on the relationship between ionic strength and deposition on to polystyrene. The effect of trypsin treatment on bacterial deposition on to polystyrene is also shown in Fig. 6. The trypsin treatment appears to have made no difference to the ionic strength dependence of $S$. salivarius deposition but markedly reduced its tendency to deposit at all ionic strengths. Trypsin treatment of $S$. mitior reduced the ionic strength dependency of its deposition without affecting its overall tendency to deposit.

The deposition of polystyrene latex spheres on to polystyrene is included in Fig. 6 for comparison. These showed a similar ionic strength dependence but less tendency to deposit than the bacteria. Streptococcus salivarius exhibited a marked reduction in deposition when deprived of its 'fuzzy coat' by trypsin treatment. This observation suggests that the fuzzy coat of the bacteria is involved in the attractive component of the interaction between the organism and the substrate. The fuzzy coat, which has been observed in vivo by Gibbons, van Houte \& Liljemark (1972) and Barnett (1973), may provide a polymer bridging means of attachment. 


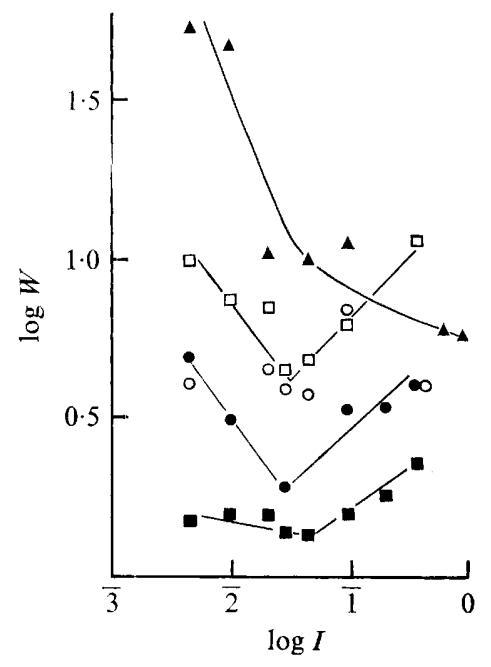

Fig. 6. Effect of ionic strength on the deposition of $S$. salivarius ( $\mathbf{\square})$, trypsin-treated $S$. salivarius $(\square), S$. mitior $(\boldsymbol{O})$, trypsin-treated $S$. mitior $(O)$ and polystyrene latex spheres $(\boldsymbol{\Delta})$ on to polystyrene on the rotating disc. Each point is the average of 8 values.

\section{DISCUSSION}

The rotating disc provides a convenient method of comparing the tendencies of different organisms to deposit on to surfaces. Providing the organisms are of similar shape and size, their collision frequency with the disc surface can be controlled by varying the speed of rotation of the disc and the bacterial suspension concentration. The tendencies for $S$. salivarius, $S$. mitior and polystyrene latex to deposit were compared by determining their collision efficiencies at different ionic strengths. The values of $W$ were calculated assuming that the Levich equation is applicable to the system.

Flocculation studies carried out by Reerink \& Overbeek (1954) showed that the flocculation of various sols could be described by $\log W$ versus $\log C$ curves which crossed the line $\log W=0$ at a critical electrolyte concentration $C_{\ell}$. At electrolyte concentrations higher than $C_{t}$ any residual repulsion between the particles was insufficient to hold them apart, and consequently every collision was successful. Under these conditions the following relationship is applicable (Visser, 1972):

$$
A=\frac{12 k T \mathrm{~d}(\log W)}{K_{\mathrm{f}} a \mathrm{~d}\left(\log C_{t}\right)}
$$

where $A$ is the Hamaker constant and $K_{\mathrm{t}}$ is the reciprocal Debye-Hückel double-layer thickness (Shaw, 1966) when $W=1$.

In the rotating disc experiments described, this condition was not achieved, although a minimum value of $\log W$ was observed for the bacteria and an inflexion was observed in the $\log W$ versus $\log C$ curve for the polystyrene latex (Figs 4 and 6). Thus there appears to be a critical electrolyte concentration, although it does not correspond to $\log W=0$. A similar relationship was obtained by Hull \& Kitchener (1969) who showed an inflexion in a $\log W$ versus $\log C$ curve describing the deposition of polystyrene latex to polyvinylformaldehyde film at $W=$ approx. 10 and $C=0.05 \mathrm{M}$. They concluded that the differences between the experimental results and theoretical predictions were due to geometrical and electrochemical heterogeneities on the surfaces involved. In the geometrical case, deposition may have occurred on to projecting parts of the disc surface rather than on to a perfect plane; in the electrochemical case, deposition may have occurred on to areas with less 
negative potential than the average value. In a similar study, Clint et al. (1973) claimed to have achieved good agreement between theory and experiment by allowing for coagulation in the dispersion during the course of the experiment. However, although the correct explanation for the difference between theory and experiment is important when calculating the interaction energies between the particles and the disc, it is less so when the method is used to compare the adhesiveness of two different organisms.

The results shown in Figs 1 to 6 illustrate three important points: (i) both organisms appear to deposit more readily than polystyrene latex to a polystyrene surface; (ii) $S$. salivarius deposits more readily to polystyrene than to glass; and (iii) the tendency of $S$. salivarius to deposit is considerably reduced if its fuzzy layer is removed. Qualitatively, the results may be discussed with respect to the attractive and repulsive interactions which occur between the particles and the disc surface. The attractive interaction is produced by charge fluctuations within the molecular structure of the two approaching surfaces, and is known as van der Waals' attraction. This is dependent on the separation distance between the two interacting surfaces and the Hamaker constant $A$.

A number of estimates (Visser, 1972) of the Hamaker constant of polystyrene generally show it to be larger than for biological cells, so it is unlikely that the van der Waals' attraction is responsible for the increased tendency for the bacteria to deposit compared with the polystyrene spheres. The repulsive interaction is more difficult to consider because of the diffuse region outside the cell wall (up to $20 \mathrm{~nm}$ thick for $S$. salivarius). Table 1 shows that the bacteria and the latex particles have similar electrophoretic mobilities. However, unless the precise value, location and distribution of the surface potentials are known the repulsive interactions cannot be accurately determined. It is possible that because of the complex nature of the bacterial surface the effective charge repulsion between the bacteria and the disc surface might be sufficiently low compared with the latex particles to explain the observed differences in deposition. In this case, the deposition of the bacteria and the latex particles could be explained in terms of van der Waals' attraction and electrostatic repulsion.

In addition to these interactions, however, a further attractive interaction may occur if the material of the fuzzy layer is capable of adsorbing to the surface of the disc. The polymer bridges so formed might be able to anchor the bacteria to the disc's surface. A similar polymer bridging mechanism was described by Fleer \& Lyklema (1974) when discussing the results of experiments concerned with the flocculation of silver iodide by polyvinylalcohol. The tendency of streptococci to deposit might therefore be due to the material of the fuzzy coat reducing the electrostatic repulsion, to the ability of this material to form polymer bridges, or possibly to a combination of both effects.

In addition to their greater tendency to deposit, the streptococci also differ from polystyrene latex in showing a maximum in their tendency to deposit which decreases with further increases in ionic strength. This may again be related to the diffuse nature of the cell wall since it does not occur with the discrete polystyrene latex particles. Again, the reduction in deposition could be due to changes in the charge distribution within the fuzzy layer, to a reduction in the tendency of the fuzzy layer to adsorb, or to a change in the conformation of the polymers within the fuzzy layer. The adsorption of $T_{2}$ phage to Escherichia coli shows a similar maximum at about $0.3 \mathrm{M}-\mathrm{NaCl}$. Brabec \& Vetterl (1967) suggested that in this case the reduction in adsorption caused by a further increase in electrolyte concentration is due to ions displacing the phage from the bacterial surface.

The difference in deposition of $S$. salivarius on to glass and polystyrene may also be due to charge or polymer adsorption. If the surface potential of glass were higher than that of the polystyrene, or the charge distribution over the surface were more even, a reduction in deposition might be expected. Similarly, if the tendency for the polymers present in the fuzzy layer to adsorb to the hydrophilic glass surface was less than that for the more hydrophobic polystyrene, a reduction in deposition would also result. An 
increased tendency of proteins to adsorb to hydrophobic surfaces compared to hydrophilic surfaces has been described by MacRitchie (1972).

Although the precise mechanism of attachment between the streptococci and the solid surfaces studied is still unclear, the results demonstrate that the rotating disc provides a convenient method of comparing the tendencies of different organisms to deposit. The results suggest that polymer bridging should be considered in addition to van der Waals' attraction and electrostatic repulsion in any explanation of bacterial adhesion. The system studied is of the simplest kind and extrapolation of the results to the situation in vivo should be made with care. In saline suspensions, $S$. salivarius exhibits a greater tendency to deposit to hard surfaces than does $S$. mitior, whereas in the oral cavity the opposite is true (Gibbons \& van Houte, 1973). This observation emphasizes the postulated influence of the extracellular diffuse layer on deposition. The nature of the suspending medium would profoundly influence the deposition of a bacterium by its interaction with the fuzzy coat. In the natural situation with a multicomponent suspending medium, the effect of each component would need to be assessed since each component would be a potentially adsorbing species to the surface, to the cell or to both. In the oral cavity, saliva is one such multicomponent suspending medium. The effect of adsorbed species on the deposition of organisms is now being studied and may help to clarify the situation.

We gratefully acknowledge the helpful discussions with Dr A. Lips, and the technical assistance of Astrid Webster, Margaret Wakeman and Jane Heathcock in preparing electron micrographs. We would also like to thank. Alok Ray for preparing the polystyrene latex.

\section{REFERENCES}

BarnetT, M. L. (1973). Adherence of bacteria to oral epithelium in vivo. Electron microscope observations. Journal of Dental Research 52, 1160.

Brabec, V. \& VetTerL, V. (1967). Adsorption of bacteriophage on bacterial surfaces and on the surface of the $\mathrm{Hg}$ dropping electrode. Biophysik 3, 361-376.

Clint, G. E., Clint, J. H., Corkhill, J. M. \& WALKER, T. (1973). Deposition of latex particles on to a planar surface. Journal of Colloid and Interface Science 44, 121-132.

Fleer, G. J. \& Lyklema, J. (1974). Polymer adsorption and its effect on the stability of hydrophobic colloids. The flocculation process as studied with the silver iodide-polyvinyl alcohol system. Journal of Colloid and Interface Science 46, 1-12.

Fletcher, M. (1976). The effects of proteins on bacterial attachment to polystyrene. Journal of General Microbiology 94, 400-404.

Gibbons, R. J. \& van Houte, J. (1973). On the formation of dental plaques. Journal of Periodontology 44, 347-360.

Gibbons, R. J., van Houte, J. \& Liljemark, W. F. (1972). Parameters that affect the adherence of $S$. salivarius to oral epithelial surfaces. Journal of Dental Research 51, 424-435.

Goodwin, J. W., Hearn, J., Ho, C. C. \& Ottewill, R. H. (1973). The preparation and characterisation of polymer latices formed in the absence of surface active agents. British Polymer Journal 5, 347-362.
Hardie, J. M. \& Bowden, G. H. (1976). Physiological classification of oral viridans streptococci. Journal of Dental Research 55, special issue A, 166-176.

Hull, M. \& Kitchener, J. A. (1969). Interaction of spherical colloidal particles with planar surfaces. Transactions of the Faraday Society 65 , 3093-3104.

Levich, V. G. (1962). Physicochemical Hydrodynamics. Englewood Cliffs, New Jersey: PrenticeHall.

MACRITCHIE, F. (1972). The adsorption of proteins at the solid/liquid interface. Journal of Colloid and Interface Science 38, 484-488.

Marshal, J. K. \& Kitchener, J. A. (1966). The deposition of colloidal particles on smooth solids. Journal of Colloid and Interface Science 22, 342-351.

Marshall, K. C., Stout, R. \& Mitchell, R. (1971). Mechanism of the initial events in the sorption of marine bacteria to surfaces. Journal of General Microbiology 68, 337-348.

ReErink, M. \& OVERBEeK, J. Th. G. (1954). The rate of coagulation as a measure of the stability of silver iodide sols. Discussions of the Faraday Society 18, 74-84.

Shaw, D. J. (1966). Introduction to Colloid and Surface Chemistry. London: Butterworth.

VISSER, J. (1972). On Hamaker constants: a comparison between Hamaker constants and Lifshitzvan der Waals constants. Advances in Colloid and Interface Science 3, 331-363. 